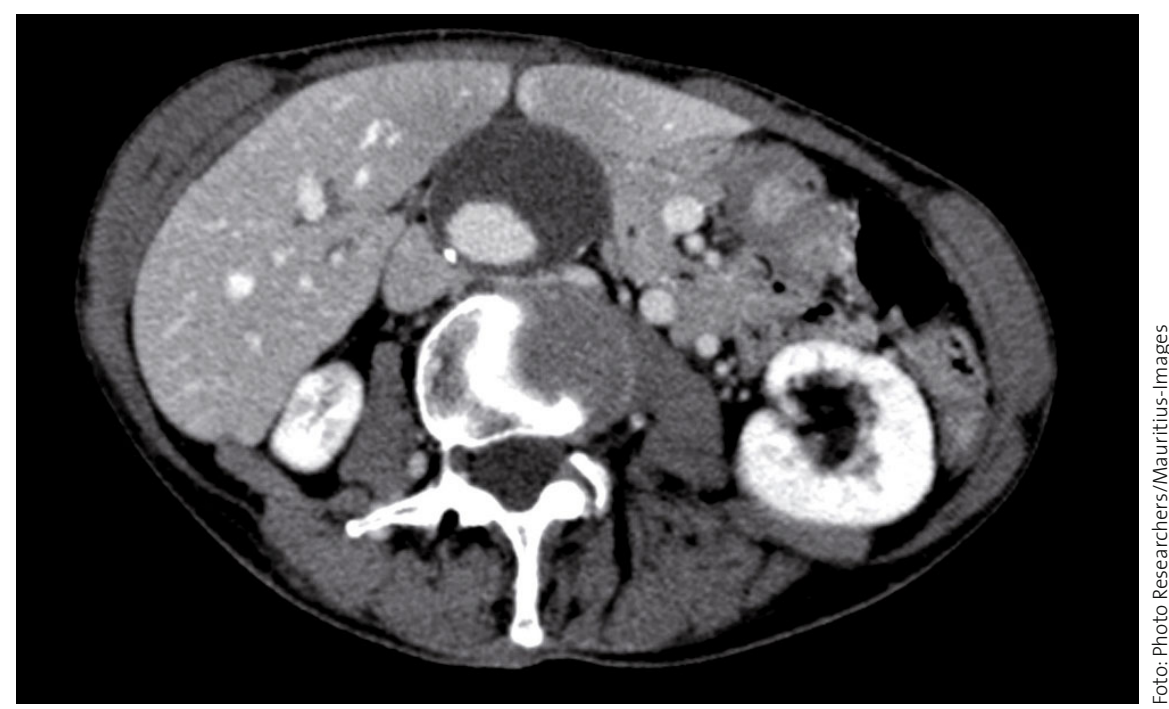

\title{
Aneurysma: Therapie offen chirurgisch oder endovaskulär?
}

Die endovaskuläre Therapie des Bauchaortenaneurysmas geht mit einer deutlich niedrigeren perioperativen Mortalität als der offene Eingriff einher, ohne dass es zu einem wesentlichen Unterschied in der Sterblichkeit im Verlauf der zwei Jahre nach dem Eingriff kommt.

— Ob die weniger invasive endovaskuläre Reparatur im Vergleich zur offenen chirurgischen Revision mit einem Bypass tatsächlich Vorteile bringt, wurde nun in einer randomisierten multizentrischen Studie an 881 Patienten im Durchschnittsalter von 70 Jahren mit einem infrarenalen abdominellen Aortenaneurysma untersucht. In randomisierter Folge unterzog sich die Hälfte der Patienten entweder einem elektiven offenen chirurgischen Eingriff oder einer endovaskulären Reparatur.

Die perioperative Mortalität lag mit $0,5 \%$ deutlich niedriger als bei offenem Vorgehen mit 3,0\%. Im Verlauf der Nachbeobachtungsperiode von zwei Jahren lag die Mortalität nach beiden Eingriffen aber vergleichbar hoch $(7,0$ vs. 9,8\%). Wie zu erwarten dauerte die offene Operation mit 3,7 Stunden deutlich länger als die endovaskuläre Reparatur mit 2,9 Stunden. Der Vorteil einer geringeren perioperativen Mortalität- und Komplikationsrate wurde nicht durch eine erhöhte Morbidität oder Mortalität im Lauf der folgenden zwei Jahre wieder wettgemacht. Dabei bestand auch kein wesentlicher Unterschied, wenn man die Patientengruppe mit einem Aneurysmadurchmesser unter 5,5 cm mit der Gruppe mit größerem Durchmesser verglich.

\section{KOMMENTAR: Zumindest bei einer} Nachverfolgungsdauer von bis zu zwei Jahren bringt der endovaskuläre Eingriff deutliche perioperative Vorteile, ohne dass sich zumindest die mittelfristige Prognose verschlechtert. Es bleibt allerdings abzuwarten, wie sich der endovaskuläre Eingriff langfristig auf den Krankheitsverlauf auswirkt. Aufgrund der bisherigen Datenlage scheint es angezeigt, hochbetagten Patienten mit hohem Operationsund Narkoserisiko eher den endovaskulären Eingriff zu empfehlen.

H. S. FÜEßL =

- F. A. Lederle et al.

Outcomes following endovascular vs open repair of abdominal aortic aneurysm. JAMA 302 (2009) 14, 1535-1542 